\title{
CrdR function in a curdlan-producing Agrobacterium sp. ATCC31749 strain
}

\author{
Xiaoqin Yu', Chao Zhang ${ }^{1}$, Liping Yang ${ }^{1}$, Lamei Zhao', Chun Lin ${ }^{1}$, Zhengjie Liu ${ }^{1}$ and Zichao Mao ${ }^{12^{*}}$
}

\begin{abstract}
Background: Agrobacterium sp. ATCC31749 is an efficient curdlan producer at low pH and under nitrogen starvation. The helix-turn-helix transcriptional regulatory protein ( $\mathrm{crdR}$ ) essential for curdlan production has been analyzed, but whether $c r d R$ directly acts to cause expression of the curdlan biosynthesis operon (crdASC) is uncertain. To elucidate the molecular function of $c r d R$ in curdlan biosynthesis, we constructed a $c r d R$ knockout mutant along with $\mathrm{pBQ} c r d R$ and $\mathrm{pBQN} c r d R$ vectors with $c r d R$ expression driven by a $T 5$ promoter and $c r d R$ native promoter, respectively. Also, we constructed a pAG with the green fluorescent protein (GFP) gene driven by a curdlan biosynthetic operon promoter ( $c r d P$ ) to measure the effects of $c r d R$ expression on curdlan biosynthesis.
\end{abstract}

Results: Compared with wild-type (WT) strain biomass production, the biomass of the crdR knockout mutant was not significantly different in either exponential or stationary phases of growth. Mutant cells were non-capsulated and planktonic and produced significantly less curdlan. WT cells were curdlan-capsulated and aggregated in the stationery phase. pBQcrdR transformed to the WT strain had a 38\% greater curdlan yield and pBQcrdR and pBQNcrdR transformed to the crdR mutant strain recovered 18\% and 105\% curdlan titers of the WT ATCC31749 strain, respectively. Consistent with its function of promoting curdlan biosynthesis, curdlan biosynthetic operon promoter ( $c r d P$ ) controlled GFP expression caused the transgenic strain to have higher GFP relative fluorescence in the WT strain, and no color change was observed with low GFP relative fluorescence in the crdR mutant strain as evidenced by fluorescent microscopy and spectrometric assay. q-RT-PCR revealed that $c r d R$ expression in the stationary phase was greater than in the exponential phase, and $c r d R$ overexpression in the WT strain increased $\operatorname{crdA}, \operatorname{crdS}$, and $\operatorname{crdC}$ expression. We also confirmed that purified crdR protein can specifically bind to the crd operon promoter region, and we inferred that $c r d R$ directly acts to cause expression of the curdlan biosynthesis operon (crdASC).

Conclusions: $C r d R$ is a positive transcriptional regulator of the crd operon for promoting curdlan biosynthesis in ATCC31749. The potential binding region of $\mathrm{crdR}$ is located within the $-98 \mathrm{bp}$ fragment upstream from the $\mathrm{crdA}$ start codon

Keywords: $c r d R$, Curdlan, Agrobacterium, Transcriptional regulator

\section{Background}

Microbes can produce diverse extracellular polysaccharides (EPS) for survival in harsh conditions [1]. Curdlan, a water insoluble $\beta$-D-1, 3-glucan, can be efficiently produced by Agrobacterium sp. ATCC31749 during stressors of low $\mathrm{pH}$ and nitrogen starvation [2-4]. Because of its special gel and immunomodulatory properties, curdlan

\footnotetext{
* Correspondence: mao2010zichao@126.com

${ }^{1}$ College of Agriculture and Biotechnology, Yunnan Agricultural University, Kunming, China

${ }^{2}$ National and Local Joint Engineering Research Center for Screening and Application of Microbial Strains, Kunming, China
}

and its derivatives can be used as food additives and in pharmaceutic products [5-7]. $\beta$-D-1,3-glucans can be synthesized by bacteria, fungi [8] and plants [9]; however, large-scale curdlan production occurs mainly via fermentation in Agrobacterium [3,10], Rhizobium strains [11] and Cellulomonas flavigena [12]. An efficient curdlanproducing strain, ATCC 31749 , whose draft genome sequence is more than $95 \%$ homologous to the Agrobacterium tumefaciens strain C58 (ATCC33970) genome, is regarded as a model organism for elucidating curdlan biosynthetic pathways and regulatory mechanisms [13,14]. Using chemical mutant selection, the curdlan biosynthesis

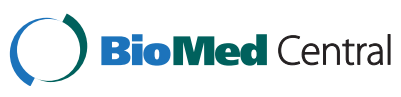

(C) 2015 Yu et al.; licensee BioMed Central. This is an Open Access article distributed under the terms of the Creative Commons Attribution License (http://creativecommons.org/licenses/by/4.0), which permits unrestricted use, distribution, and reproduction in any medium, provided the original work is properly credited. The Creative Commons Public Domain Dedication waiver (http://creativecommons.org/publicdomain/zero/1.0/) applies to the data made available in this article unless otherwise stated. 
operon ( $c r d$ ) was found to contain $c r d A, c r d S$, and $c r d C$ genes in the ATCC31749 strain [15-17]. Many cultivating conditions including low $\mathrm{pH}$ [18], limited nitrogen [19], high dissolved oxygen [20] and adding uracil or cytosine and phosphate salts [21-23] influence curdlan biosynthesis and accumulation. However, how curdlan biosynthesis gene expression is regulated is unclear.

ATCC31750, a mutant strain derived from ATCC31749, had significantly altered intracellular proteins with changes in $\mathrm{pH}$. Specifically, at pH 5.5 (compared to 7.0), key enzymes of curdlan biosynthesis, such as the catalytic subunit of $\beta$-1,3-glucan synthase $(r d S)$, UTP-glucose-1-phosphate uridylytransferase (gall), and phosphoglucomutase (pgm) were increased 10, 3, and 17 times, respectively [18]. Intracellular $\mathrm{pH}$ changes may activate synthesis of a cellular stringent response signal (p)ppGpp to alter formation of acidocalcisome, which helps maintain intracellular $\mathrm{pH}$ and ion homeostasis [24]. To learn how low pH affects curdlan biosynthesis in an ATCC31749 strain, we analyzed genomic sequences of ATCC31749 (access No: AECL01000001-AECL01000095) and that of Sinorhizobium meliloti (access No: NC_003047), which is an acid-tolerant, symbiotic nitrogen-fixing strain [25] using BLAST alignment. We found a transcriptional regulator, PhrR (access No: NC_003047.1 (445435-445854), expression of this gene increased 5-6 times under conditions of low pH (pH 6.2) in S. meliloti [26]. The PhrR gene has a homologous counterpart, AGRO_0435, in ATCC31749. Both PhrR and AGRO_0435 are helix-turn-helix transcriptional factors of the XRE-family, which includes HipB of Escherichia coli (E coli), CH00371 of Rhizobium Leguminosarum (R. Leguminosarum), and PraR of Azorhizobium Caulinodans (A. caulinodans) (Additional file 1) [27-35]. The existence of an essential curdlan production regulatory locus other than the crd operon-locus II-was suggested by Stasinopoulos's group [15] DNA sequencing confirmed that the locus II gene encodes a helixturn-helix transcriptional regulatory protein, crdR, and that AGRO_0435 is the $c r d R$ gene [26], Unfortunately, whether crdR acts directly to regulate $\operatorname{crdASC}$ expression is unclear, so we investigated the role of $\operatorname{crdR}$ on crdASC transcriptional activation.

\section{Methods}

\section{Bacterial strains and vectors used}

Strains and vectors used are listed in Table 1. E. coli strains TG1 and BL21 used for cloning and expression were grown in Luria-Bertani broth (LB). The Agrobacterium sp. ATCC31749 strain was cultivated in LB for growth and for curdlan production, in curdlan-producing medium ([w/v], $5 \%$ sucrose, $0.005 \%$ yeast extract, $0.5 \%$ citric acid, $0.27 \% \mathrm{~K}_{2} \mathrm{HPO}_{4}, 0.17 \% \mathrm{KH}_{2} \mathrm{PO}_{4}, 0.01 \% \mathrm{MgSO}_{4}$,

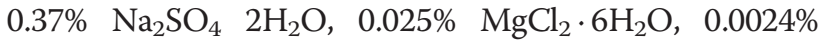
$\mathrm{FeCl}_{3} \cdot 6 \mathrm{H}_{2} \mathrm{O}, 0.0015 \% \mathrm{CaCl}_{2} \cdot 2 \mathrm{H}_{2} \mathrm{O}$, and $0.001 \% \mathrm{MnCl}_{2}$.
Table 1 Bacterial strains and plasmids used in this study

\begin{tabular}{|c|c|c|}
\hline Strain/plasmids & Description & Sources \\
\hline \multicolumn{3}{|l|}{ Strains } \\
\hline E.coli BL21 & $\begin{array}{l}\operatorname{Res}^{-} \text {Mod }^{-} \text {ompT (DE3 with } T 7 \text { pol) } \\
\text { (pLysS with } T 7 \text { lysozyme; }\left(m^{\prime} \text { ) }\right. \\
\text { Novagen }\end{array}$ & Lab stock \\
\hline E.coli TG1 & Cloning host & TaKaRa \\
\hline ATCC31749 & $\begin{array}{l}\text { Curdlan-producing Agrobacterium sp. } \\
\text { (wild-type strain) }\end{array}$ & ATCC \\
\hline ATCC31749 $\Delta \mathrm{crdR}$ & $\begin{array}{l}\text { ATCC } 31749 \text { mutant with gene } \\
\text { knockout of } c r d R\end{array}$ & This study \\
\hline \multicolumn{3}{|l|}{ Plasmids } \\
\hline pEGFP & GFP expression vector & Clontech \\
\hline pQE81L & Expression vector, $A m p^{R}$ & Qiagen \\
\hline pBBR122 & Gram-negative broad host vector & MoBiTec \\
\hline pEX18Gm & Expression vector carrying $\mathrm{sacB}^{\mathrm{R}}, \mathrm{Gm}^{\mathrm{R}}$ & {$[36]$} \\
\hline $\mathrm{pBQ}$ & $\begin{array}{l}\text { Vector derived from both pQE80 and } \\
\text { pBBR122 }\end{array}$ & {$[37]$} \\
\hline pUC19 & Cloning vector & TaKaRa \\
\hline pUC19T-crdR & Suicide vector for $c r d R$ knock-out & This study \\
\hline pBQcrdR & $\begin{array}{l}\text { Expression vector with } T_{5} \text { driving } \\
c r d R \text { expression }\end{array}$ & This study \\
\hline pBQNcrdR & $\begin{array}{l}\text { Expression vector with } c r d R P \text { driving } \\
c r d R \text { expression }\end{array}$ & This study \\
\hline pAG & $\begin{array}{l}\text { Expression vector with GFP driven by } \\
\text { crd promoter }\end{array}$ & This study \\
\hline pMD18-T(crdA) & Derivative of pMD18-T with part of $\mathrm{crdA}$ & This study \\
\hline pMD18-T(crdS) & Derivative of pMD18-T with part of crdS & This study \\
\hline pMD18-T(crdC) & Derivative of pMD18-T with part of $\mathrm{crdC}$ & This study \\
\hline pMD18-T(crdR) & Derivative of pMD18-T with part of $\mathrm{crdR}$ & This study \\
\hline
\end{tabular}

$4 \mathrm{H}_{2} \mathrm{O}$ ). Culture $\mathrm{pH}$ for strain growth was maintained at 7.0 and lowered to 5.5 immediately for curdlan production in a curdlan-producing medium [36]. Primers for PCR amplification designed by DNAMAN software and synthesized by Sangong Biotech (Shanghai, China) are listed in Table 2.

\section{Knockout of the CrdR (AGRO_0435 gene)}

For AGRO_0435 gene knockout, a 2,533 bp fragment of the target gene (AGRO_0435) with up- and down-stream flanking sequences was PCR cloned using primers crdRup-1 and crdRdown-2. The amplified fragment, double digested with both SacI and HindIII, was inserted into the same sites of pUC19 to obtain the pUCcrdR flanking. The gentamicin $(\mathrm{Gm})$ resistance gene expression cassette, obtained by PCR amplification with primers (Gm-1 and Gm-2) from pEX18Gm [37], was double digested with BamHI and SalI and then inserted into the same sites of the pUCcrdR flanking to obtain the suicide knockout vector pUC19T-crdR (Figure 1A). Knockout plasmids were transformed with electroporation using an 
Table 2 Primers used in this study

\begin{tabular}{|c|c|c|c|}
\hline Primer name & Oligonucleotide & Product length & Product name \\
\hline $\operatorname{crdPG-1}$ & GTACTCGAGATTGTCGGCAGTCCAG & 607 & $\operatorname{crdP}$ \\
\hline $\operatorname{crdPG-2}$ & AGCTCCTCGCCCTTGCTCACCATGAAATCAACTCCTCTGT & & \\
\hline GFP-1 & ACAGAGGAGTTGATTTCATGGTGAGCAAGGGCGAGGAGCT & 746 & GFP \\
\hline GFP-2 & CGCGGATCCTTACTTGTACAGCTCGTCCATG & & $\operatorname{crdP}$ \\
\hline $\mathrm{crdP}-1$ & TCACCAACACCAACTCTGGA & & \\
\hline crdP-2 & CATGAAATCAACTCCTCTGT & 607 & $\operatorname{crdP}$ \\
\hline crdP142-1 & ATCGTCAGATGCCTATTTGT & 537 & $\operatorname{crdP}$ \\
\hline crdP108-1 & AAATTAGTTAATGCAAT & 503 & $\operatorname{crdP}$ \\
\hline crdP98-1 & TTAATGCAATITTACTATGTT & 493 & $\operatorname{crdP}$ \\
\hline crdP53-1 & CCATTTCAATACTGCGGGAGG & 448 & $\operatorname{crdP}$ \\
\hline crdP13-1 & AGGAGTTGATTTCATGCTGTT & 408 & $\operatorname{crdP}$ \\
\hline crdP1-1 & ATGCTGTTCCGCAATAAG & 395 & $\operatorname{crdA}$ \\
\hline $\operatorname{crdA395-2}$ & TCGGTCCGCAGCAGCAAAG & & \\
\hline$q-c r d A-1$ & CAAGGCATAAGCGAAGACATC & 227 & $\operatorname{crdA}$ \\
\hline$q-\operatorname{crd} A-2$ & CTCCGTGTTTCAAGTGTGGTC & & \\
\hline$q-c r d S-1$ & AACCTGACGATTGCGATTGGG & 179 & $\operatorname{crds}$ \\
\hline$q-c r d S-2$ & GTGTAGCACCAGAGCGTTTCG & & \\
\hline$q-\operatorname{crd} C-1$ & GTTCGGTCAGGATGCTCAAC & 248 & $\operatorname{crdC}$ \\
\hline$q-\operatorname{crdC}-2$ & GCCAAAGTTCGGAATCAATG & & \\
\hline $\operatorname{crdR-1}$ & GCCAGATCTATGACCGAGAATAAGAAAAAGCCT & 437 & $\operatorname{crdR}$ \\
\hline $\operatorname{crdR}-2$ & TTGAGCTCTTACTCGGCGTCGCCTTCG & & \\
\hline NcrdR-1 & ACACTCGAGATACACCCGGTCCCTACCAGCATT & & $\operatorname{crdR}$ \\
\hline NcrdR-2 & TTTGAGCTCCTTGTCCTTCTTCAGAAGCGTGT & 1302 & $\operatorname{crdR}$ \\
\hline$q-\operatorname{crd} R-1$ & TCGGAATGAGCCAGGAGAAGC & 238 & $\operatorname{crdR}$ \\
\hline$q-c r d R-2$ & TCAGCCGAGGACAGAAAGTCG & & \\
\hline crdRup-1 & TCTGAGCTCTTCGGCGTTTCGGAATGGTTG & 2533 & $\operatorname{CrdR}$ \\
\hline crdRdown-2 & CAACACAAGCTTAACCGTCACCTGGCTCTTGGCA & & \\
\hline crdRcheckGm-2 & TTGGGCATACGGGAAGAAGT & 558 & Gm \\
\hline crdRcheckGm-1 & CGGCTGATGTTGGGAGTAGG & 577 & \\
\hline Rep-Kan-1 & ACGCGTCGACCTTGCCAGCCCGTGGATAT & 3266 & $\mathrm{Km}$ \\
\hline Rep-Kan-2 & ACGCGTCGACTCTGTGATGGCTTCCATGTC & & \\
\hline Gm-1 & ATAGTTGTCGAGATATTCACTAGTCGTCAGGTGGCACTTITCG & 1302 & Gm \\
\hline Gm-2 & CGCGGATCCGCTCTGCTGAAGCCAGTTAC & & \\
\hline celAP1 & ACCCGGTCTATCCCATGA & & \\
\hline celAP-2 & CATCCAGAAACTITCCGT & 441 & CelAP \\
\hline relAP-1 & CCAGATTTCTCAAGGGTC & & \\
\hline RelAP-2 & CATCATGCGATATTCCACA & 443 & relAP \\
\hline crdRP-1 & GCGGCGATCCTAAATGTGAC & & \\
\hline $\operatorname{crdRP}-2$ & CATGCGGTCCTGACACTCG & 466 & $\operatorname{crdRP}$ \\
\hline crdSP-1 & TCGGTCGGCACATGGGTCAAT & & \\
\hline crdSP-2 & CATCGCCCTAACCTCGCAGT & 446 & crdSP \\
\hline
\end{tabular}

Eppendorf micropulser. Knockout mutants, selected by screening on LB-agar plates containing $24 \mu \mathrm{g} / \mathrm{mL}$ gentamicin, were confirmed with PCR amplification with 3 pairs of primers including crdR-1 and crdR-2, crdR-1 and crdRcheckGm-2, and crdRcheckGm-1 and crdR-2 (Table 2). 

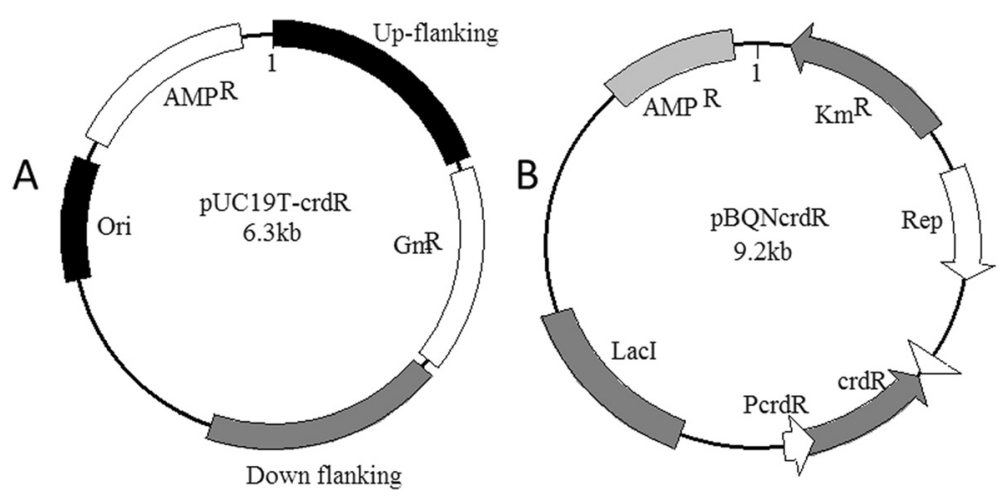

Figure 1 Maps of pUC19T-crdR (A) and pBQNcrdR (B).

\section{Construction of $\mathrm{pBQ} c \mathrm{ddR}$ and $\mathrm{pBQNcrdR}$ for homogenous AGRO_0435 expression}

A 437-bp full-length coding region of $c r d R$ was amplified with PCR using the primer pairs crdR-1 and crdR-2 (Table 2) with genomic ATCC31749 DNA. The amplified $c r d R$ fragment, digested with $B a m H I$ and SacI, was ligated into the $\mathrm{pBQ}$ vector [38], creating the $\mathrm{pBQ}$ crdR vector (Table 1). To construct the vector for $c r d R$ expression driven by its native promoter of $\mathrm{crdR}$, an AGRO_0435 fragment with up- and down-stream flanking sequences was PCR cloned using primers NcrdR-1 and NcrdR-2 (Table 2) The obtained 1,302 bp PCR fragment which was double digested with both $\mathrm{SacI}$ and $\mathrm{XhoI}$ was inserted into SalI and sacI sites of pBQ to create pBQNcrdR (Table 1, Figure 1).

\section{Construction of pAG vector with GFP expression driven by the $c r d$ operon promoter}

The predicted $c r d$ promoter $(c r d P)$, which is a 607-bp fragment upstream from the start codon of $c r d A$ (ATG), was amplified from genomic ATCC31749 DNA with primers crdAPG-1 and crdAPG-2 (Table 2). The GFP code sequence was amplified with primers GFP-1 and GFP-2 (Table 2) from plasmid pEGFP (Clontech, Mountain View, $\mathrm{CA}$ ) and the two fragments were fused via PCR amplification. The resultant fused fragment, digested with $\mathrm{XhoI}$ and $B a m H I$, was inserted into the same sites of plasmid pQE81L to yield pQEAG. After digestion with SalI, the fragment containing the gram-negative broad host replicating origin and the kanamycin (Kan) resistant gene amplified from pBBR122 (Table 1) with primers pairs Rep-Kan-1 and Rep-Kan-2 (Table 2), was inserted into the XhoI site of pQEAG to yield pAG (Table 1).

\section{Curdlan fermentation and yield analysis}

A two-step fermentation protocol was used to measure curdlan yields. In brief, ATCC 31749 and modified strains were inoculated into test tubes containing $5 \mathrm{~mL} \mathrm{LB}$ and grown overnight at $30^{\circ} \mathrm{C}$ with 200 revolutions per minute (rpm). About $2 \mathrm{~mL}$ each of the seed cultures (SC) were transferred into 500-mL flasks containing $100 \mathrm{~mL} \mathrm{LB}$ with or without IPTG (final concentration $0.5 \mathrm{mM}$ ) at $25^{\circ} \mathrm{C}$, $200 \mathrm{rpm}$ for $4 \mathrm{~h}$. Cells were collected by centrifugation $\left(1000 \times \mathrm{g}\right.$ for $\left.10 \mathrm{~min}, 4^{\circ} \mathrm{C}\right)$ and cell pellets were added to $125 \mathrm{~mL}$ curdlan-producing medium in a $500-\mathrm{mL}$ flask which was shaken at $200 \mathrm{rpm}$. Every $24 \mathrm{~h}$ for 5 days reaction, $15-\mathrm{mL}$ samples were taken from the culture mixture and samples were centrifuged at $8,000 \times \mathrm{g}$ for $5 \mathrm{~min}$ to collect pellets. Pellets containing both cells and curdlan were resuspended in $15 \mathrm{~mL} \mathrm{NaOH}$ solution $(1 \mathrm{~mol} / \mathrm{L})$ for $2 \mathrm{~h}$. Cells pellets were separated by centrifugation at $8,000 \times \mathrm{g}$ for $5 \mathrm{~min}$ and resulting curdlan was precipitated by the addition of $2.0 \mathrm{~mol} / \mathrm{L} \mathrm{HCl}$ and the $\mathrm{pH}$ was adjusted to 6.5. Curdlan was recovered by centrifugation, washed, and dried to a constant weight in an oven $\left(80^{\circ} \mathrm{C}\right)$.

$c r d R, c r d A, c r d S$, and $c r d C$ expression analysis using q-RT-PCR Total RNA was extracted with an EasyPure RNA Kit (TransGen Biotech, Beijing, China), according to the manufacturer's protocol. The quality and quantity of the extracted RNA was measured using an Ultrospec 2100 spectrophotometer (Amersham Biosciences, Pittsburgh PA, USA) at $260 \mathrm{~nm}$. cDNA synthesis was performed with a PrimerScript RT reagent Kit (TaKaRa, Dalian, China) according to the manufacturer's instruction by using a 6-bp random primers set. Selected fragments of $\operatorname{crdR}, \operatorname{crdA}$, $c r d S$, and $c r d C$, which were amplified with primers qcrdR1 \& qcrdR-2, qcrdA-1 \& qcrdA-2, qcrdS-1 \& qcrdS-2, and qcrdC-1 \& qcrdC-2 (Table 2), were ligated into pMD18-T vectors respectively. Then, using those constructs as standard copies, q-RT-PCR quantification was performed using an Applied Biosystems 7500 fast realtime PCR system (Applied Biosystems, Grand Island, NY ) with SYBR Premix E*TaqII (TaKaRa). All samples were run in triplicate [39]. 


\section{GFP expression}

The constructed vector (pAG) was transformed into both wild-type ATCC31749 and a crdR mutant (ATCC3174 $9 \Delta c r d R)$. Transformed bacterial cells were grown in LB for $12 \mathrm{~h}$ and curdlan-producing medium for $72 \mathrm{~h}$ at $30^{\circ} \mathrm{C}$. GFP expression was observed under an optical microscope (Zeiss Observer Berlin, Germany), equipped with epi-fluorescence. Simultaneously, with excitation of 450$490 \mathrm{~nm}$ light, Green fluorescence of GFP was measured by a fluorospectrophotometer F97Pro (FProd, Shanghai, China) to collect the data of the emission spectrum and relative fluorescence of cells harvested from both bacterial cell -growing and curdlan-producing phases.

\section{Expression and purification of 6 His-tagged crdR protein} 6-His-tagged crdR was expressed in E. coli BL21 through pBQcrdR transformation. The resultant strain grew at $37^{\circ} \mathrm{C}$ in $\mathrm{LB}$ medium $\left(\mathrm{OD}_{600} \mathrm{~nm}=0.5-0.6\right)$, and crdR protein expression was induced by adding IPTG (final concentration $=0.5 \mathrm{mM}$ ). The culture was shaken at $30^{\circ} \mathrm{C}$ for $4 \mathrm{~h}$ at $220 \mathrm{rpm}$. Cells were harvested by centrifugation were immediately extracted or frozen at $-80^{\circ} \mathrm{C}$ until they were used. 6-His-tagged protein was purified by affinity chromatography using One-Step His-Tagged Protein Miniprep Pack (TIANDZ, Shanghai, China) according to the manufacturer's instruction. Purified crdR dissolved in elution buffer was dialyzed with dialysis buffer $(100 \mathrm{mM}$ KAc, $1 \mathrm{mM} \mathrm{CaCl}$, $1 \mathrm{mM} \mathrm{MgAc}, 1 \mathrm{mM}$ EDTA, and $1 \mathrm{mM}$ dithiothreitol, $10 \%$ glycerol) overnight in a semipermeable membrane. Protein concentration was measured using an improved Coomassie assay with bovine serum albumin (BSA) as standard.

\section{DNA binding analysis of CrdR by EMSA}

DNA fragments containing various lengths of the crd promoter (crdP, crdP142, crdP108, crdP98, crdP53, crdP13, and $c r d P 1)$ and $\sim 450$ bp upstream of the start codon (ATG) of $c r d R$ (relA(AGRO_1479) celA (AGRO_4469), and crdS (AGRO_1848) named relAP celAP and crdSP were obtained by PCR amplification with primers listed in Table 2 respectively, those fragments were purified with a DNA gel extraction kit (Sangon Biotech) respectively according to the manufacturer's protocol. A electrophoretic mobility shift (EMSA) binding assay was performed as previously described with slight modifications [40]. Briefly, $10 \mu \mathrm{L}$ of $0.25-0.50 \mathrm{mg} / \mathrm{mL}$ purified His-tagged crdR in $4 \times$ EMSA buffer (15 mM HEPES, $100 \mathrm{mM} \mathrm{NaCl}, 1 \mathrm{mM}$ EDTA, $5 \mathrm{mM} \mathrm{MgCl}_{2}, 10 \%$ glycerol, $1 \mu \mathrm{g} / \mathrm{mL}$ poly $\mathrm{dI}-\mathrm{dC}$ ) was incubated with $10 \mu \mathrm{L}$ of different purified target DNA fragments $(0.5 \mu \mathrm{M})$ in $\mathrm{ddH}_{2} \mathrm{O}$ at room temperature for $30 \mathrm{~min}$. DNA-protein complexes were loaded onto a $2 \%$ agarose gel and separated at $80 \mathrm{~V}$ for $1.5 \mathrm{~h}$, and the gel was stained with SYBR Green I and visualized with a UV trans-illuminator (Upland, CA).

\section{Results}

crdR knockout mutant construction and phenotypes

The $c r d R$ knockout mutant was constructed via homologous recombination by transformation of the suicide plasmid, pUC19T-crdR (Figure 1A). After strains were selected on gentamicin $(\mathrm{Gm})$ resistant LB plates, knockout mutants were confirmed with PCR amplification (Figure 2). Compared with wild-type (WT) ATCC31749, which is capsulated in the stationary phase, the $c r d R$ knockout strain (ATCC31749 $\Delta c r d R$ ) produced less curdlan (Figures 3 and 4) leading to motile and non-capsulated planktonic forms (Figure 3) in both exponential and stationary phases. $c r d R$ expression driven by promoters of $T 5$ and native $c r d R$ in both ATCC31749 and ATCC31749 $\Delta c r d \mathrm{R}$ strains, respectively, were obtained by transforming the constructs of pBQcrdR and pBQNcrdR (Figure 1). The ATCC31749 $\Delta c r d R / p B Q N c r d R$ strain recovered its curdlan capsulated form of ATCC31749 (Figure 3D and 3E). With a two-step flask-shaking process, curdlan production over 5 days $[18,19]$ in 5 cultivated strains-ATCC31749, ATCC31749/pBQcrdR, ATCC31749 $\Delta c r d R$, ATCC31749 $\Delta$ $c r d R / \mathrm{pBQ} c r d R$ and ATCC31749 $\Delta c r d R / \mathrm{pBQNcrdR}$-was compared to assess biomass accumulation and curdlan yields. Data show that (Figure $4 \mathrm{~B}$ ) the biomass of ATCC31749 $\Delta c r d R$, ATCC31749/pBQcrdR and ATCC3 $1749 \Delta c r d R / p B Q N c r d R$ were higher and that ATCC31 $749 \Delta c r d R / p B Q c r d R$ had less accumulation than WT ATCC 31749 during cultivation days 2 to 4 . By the $5^{\text {th }}$ day, however, cell biomasses of all strains were similar.

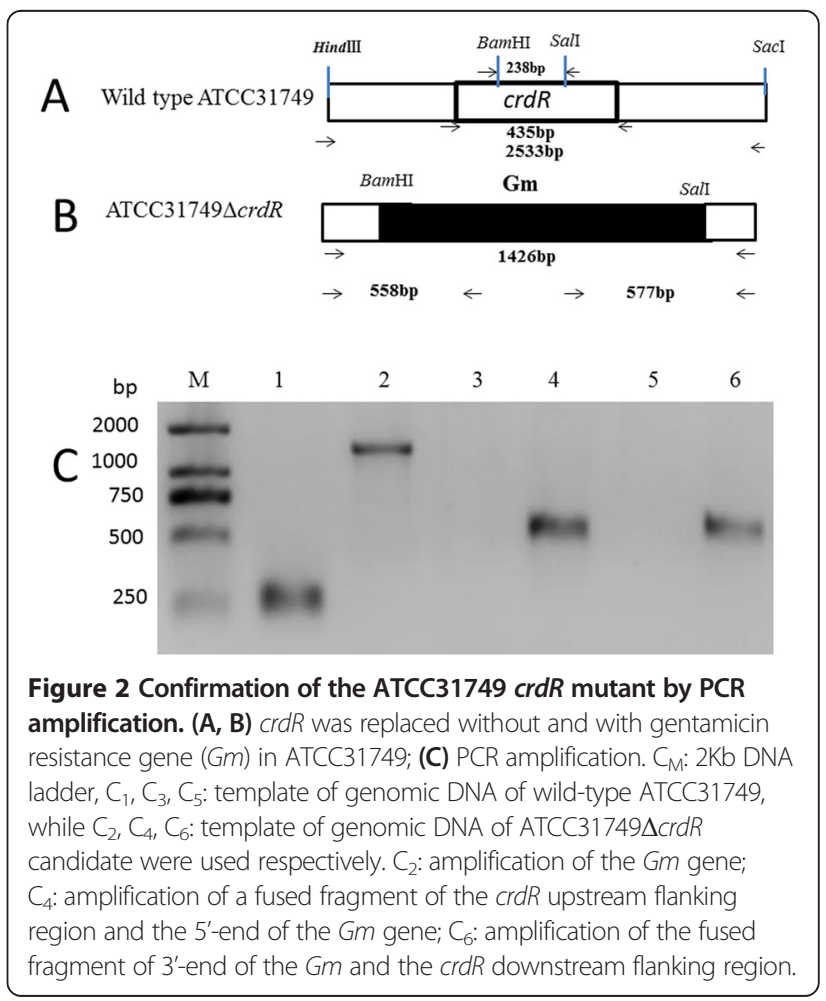




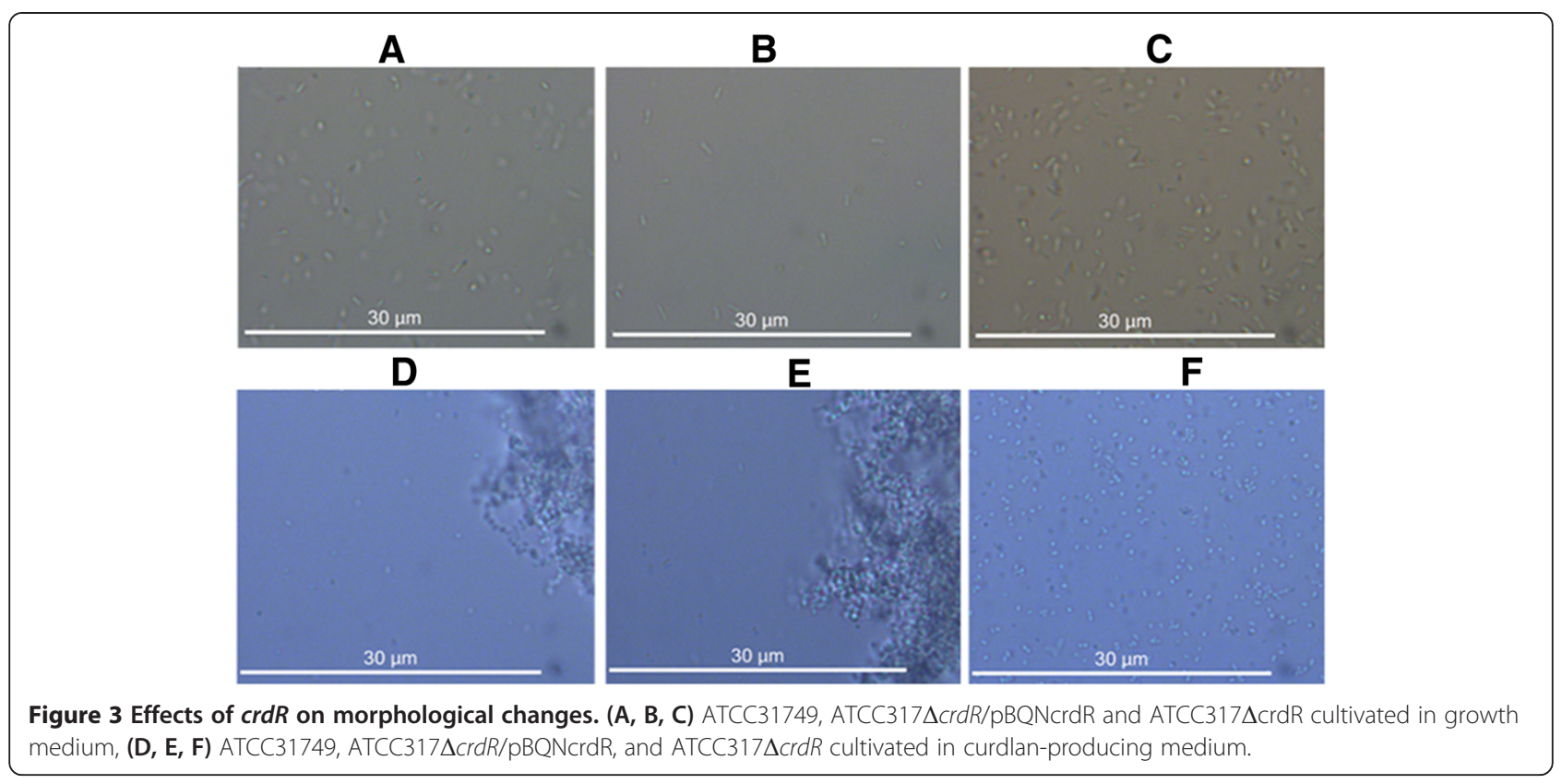

Curdlan yields for ATCC31749, ATCC31749/pBQcrdR, ATCC31749 $\Delta c r d R$, ATCC31749 $\Delta c r d R / \mathrm{pBQ}-\mathrm{crdR}$ and ATCC31749 $\Delta c r d R / p B Q N c r d R$ were $5.66 \mathrm{~g} / \mathrm{L}, 7.80 \mathrm{~g} / \mathrm{L}$, $0.007 \mathrm{~g} / \mathrm{L}, 1.13 \mathrm{~g} / \mathrm{L}$, and $5.91 \mathrm{~g} / \mathrm{L}$, respectively (Figure 4A). Curdlan yield for the $c r d R$ overexpressed strain (ATC C31749/pBQcrdR) was 38\% greater than that of WT. $c r d R$ controlled by the $T 5$ promoter in ATCC $31749 \Delta \mathrm{crdR} /$ pBQcrdR could synthesize curdlan, but yields only reached $18 \%$ of WT yields; however $c r d R$ controlled by its native promoter in ATCC $31749 \Delta c r d R / \mathrm{pBQ} c r d R$ recovered curdlan yields of $c r d R$ knockout. Low curdlan yields caused by expression of $\mathrm{crdR}$ controlled with $T 5$ promoter suggests a more complex regulatory mechanism of $c r d R$ expression in the strains. Judged from these data, our observation of crdR is consistent with reports to suggest that crdR is an important regulator of curdlan biosynthesis $[15,26]$.

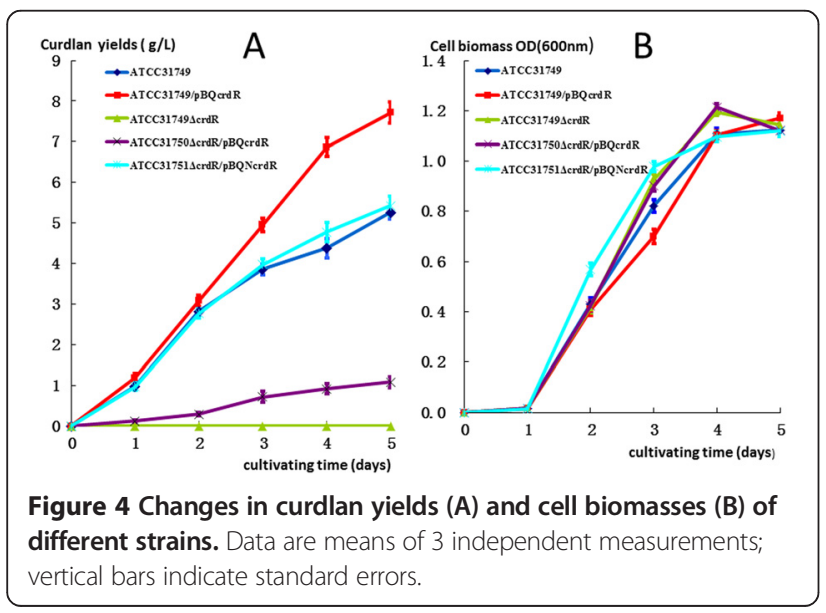

Expression of curdlan biosynthesis genes responding to crdR overexpression

Because $c r d R$ is an important regulator of curdlan biosynthesis (Figures 3 and 4), we investigated whether crdR activates expression of $c r d$ operon genes. q-RTPCR analysis was used to evaluate the effects of $c r d R$ on $\operatorname{crdA}, \operatorname{crdS}$, and $\operatorname{crdC}$ (genes of the curdlan biosynthetic operon) mRNA. Stationary phase cells favoring curdlan biosynthesis were compared with exponential phase cells favoring cell growth, and $c r d R$ native expression was found to be 29.2 copies/ng total RNA in the stationery phase and 14.0 copies/ng total RNA in the exponential phase in ATCC31749/pBQ. Correspondingly, expression of $\operatorname{crdA}, \mathrm{crdS}$, and $\mathrm{crdC}$ was at least 10 times greater in the stationary phase compared to the exponential phase (Table 3). mRNA of $c r d R$ in ATCC31749/pBQcrdR, induced by $0.5 \mathrm{mM}$ isopropyl $\beta$-D-1-thiogalactopyranoside (IPTG) at $30^{\circ} \mathrm{C}$ for $2 \mathrm{~h}$, was significantly increased compared to that in ATCC31749/pBQ. Corresponding mRNA of $c r d A, c r d S$ and $c r d C$ in ATCC31749/pBQcrdR were more than twice greater when strains were cultivated in both growth and fermentation media. Data confirmed that $c r d R$ promotes curdlan production via activating expression of $c r d$ operon genes (Table 3).

GFP expression controlled by the $c r d$ operon promoter ( $c r d P$ ) To confirm the effect of $c r d R$ on $c r d$ operon gene expression, a shuttle vector of pAG bearing GFP driven by the crdP was constructed. pAG was transformed into both ATCC31749 and its crdR mutant strain of ATCC317 $49 \Delta c r d R$. Data indicate that green fluorescence was undetectable by fluorescent microscopy in the $c r d R$ mutant strain. In contrast, strong green fluorescence was visible in 
Table 3 The expressions of the crdgenes quantified by q-RT-PCR*

\begin{tabular}{lllll}
\hline & ATCC31749/pBQ(E) & ATCC31749/pBQcrdR(E) & ATCC31749/pBQ(S) & ATCC31749/pBQcrdR(S) \\
\hline crdA & $0.2373 \pm 0.0635$ & $1.0944 \pm 0.0956$ & $244.2038 \pm 18.3645$ & $923.5690 \pm 29.6543$ \\
crdS & $0.4050 \pm 0.1022$ & $2.7739 \pm 0.1185$ & $86.7645 \pm 4.2966$ & $176.6229 \pm 9.5607$ \\
crdC & $0.4972 \pm 0.9503$ & $2.2787 \pm 0.1263$ & $4.5239 \pm 0.3371$ & $14.5744 \pm 1.0027$ \\
crdR & $14.9112 \pm 1.0359$ & $654.8556 \pm 20.4256$ & $29.2068 \pm 1.8553$ & $193.1226 \pm 11.2094$ \\
\hline
\end{tabular}

*The data are means of three independent determinations, and the unite of value is copies per ng total RNA(copies/ng RNA) E: strain in exponential phase, S:strain in stationary phase.

the WT ATCC31749 strain grown in fermentation media (Figure 5A and 5B). Also, the ATCC31749/pAG strain was curdlan capsulated whereas ATCC31749 $\Delta c r d R /$ pAG was non-capsulated and motile (Figure 5C and 5D). GFP expression detected by spectrophotometry was consistent with microscopic observations that the relative GFP florescence in ATCC31749/pAG exceeded that in ATCC $31749 \Delta \mathrm{crdR} / \mathrm{pAG}$ at both exponential and stationary phases (Figure 5D). Because the GFP expression pattern represents expression profiles of $c r d A, c r d S$ and crdC in engineered strains of both ATCC31749/pAG and ATCC31749 $\Delta c r d R / p A G$, the effects of $c r d R$ expression on GFP expression in those strains indicated that $c r d R$ might directly or indirectly interact with the $c r d$ operon promoter to regulate expression(s) of curdlan synthetic gene(s). Interestingly relative GFP florescence of exponential phase ATCC $31749 \Delta c r d R / p A G$ was less than that measured in the stationary phase, suggesting that $\operatorname{crdR}$ may synergistically cooperate with other regulators to control $c r d$ operon gene expression.

crdR binding with' different crd operon promoter regions Bioinformatic analysis of deduced amino acid sequences indicates that crdR has a conserved DNA-binding motif of a helix-turn-helix domain. To confirm that crdR protein can directly interact with the $\mathrm{crd}$ operon promoter region, with BSA as a negative protein control, DNAbinding analysis was performed with an electrophoretic mobility shift assay (EMSA) with amino terminal 6-Histagged crdR and DNA fragments containing its putative binding sites. 6 His-tagged $\mathrm{crdR}$ protein was expressed and purified into a single band $(15 \mathrm{kDa})$ with SDS-PAGE from $E$. coli $\mathrm{Bl} 21$ which was transformed with $\mathrm{pBQ}$ crdR (Figure 6B). The various crdR putative binding fragments, including serial regions of the $c r d$ promoter ranging from $607 \mathrm{bp}$ upstream from ATG of $c r d A$ fused with or without of 395 bp downstream of ATG of $c r d A$ (Figure 6A) and about 450 bp upstream from ATG of $c r d R$, (p) ppGpp synthetase (relA), cellulose synthase catalytic subunit (celA) and curdlan synthase catalytic subunit ( $c r d S)$, were amplified by PCR with the genomic DNA of ATCC31749 as a template (Figure 6E). Data indicate that 6 His-tagged crdR protein cannot specifically bind 450 bp upstream of ATG of $c r d R, \operatorname{rel} A, \operatorname{cel} A$, and $c r d S$, but can bind to different promoter regions of the $c r d$ operon (Figure 6C and 6D). To locate crdR binding site(s) on the $c r d$ operon promoter, 607 bp upstream from ATG of the $\operatorname{crdA}$ sequence which can successfully drive GFP expression in the stationary phase (Figure 5), was chosen and the fragment was shorted to $98 \mathrm{bp}$ upstream from the ATG of $\operatorname{crdA}$ to measure binding abilities to crdR. Data indicate that those sequences did not reduce binding to $\mathrm{crdR}$ as evidenced by a band shift in EMSA by mixing with or without crdR (Figure 6C). Then, the -98 bp fragments upstream from the $\operatorname{crd} A$ start codon was focused for further analysis by continual shortening to $13 \mathrm{bp}$ upstream of ATG of $\operatorname{crdA}$. Using the $395 \mathrm{bp}$ coding sequence of $\operatorname{crdA}$ from 1 to $395 \mathrm{bp}$ and BSA as a negative DNA and protein control, respectively, fragment mobility shifts containing $-98,-53$

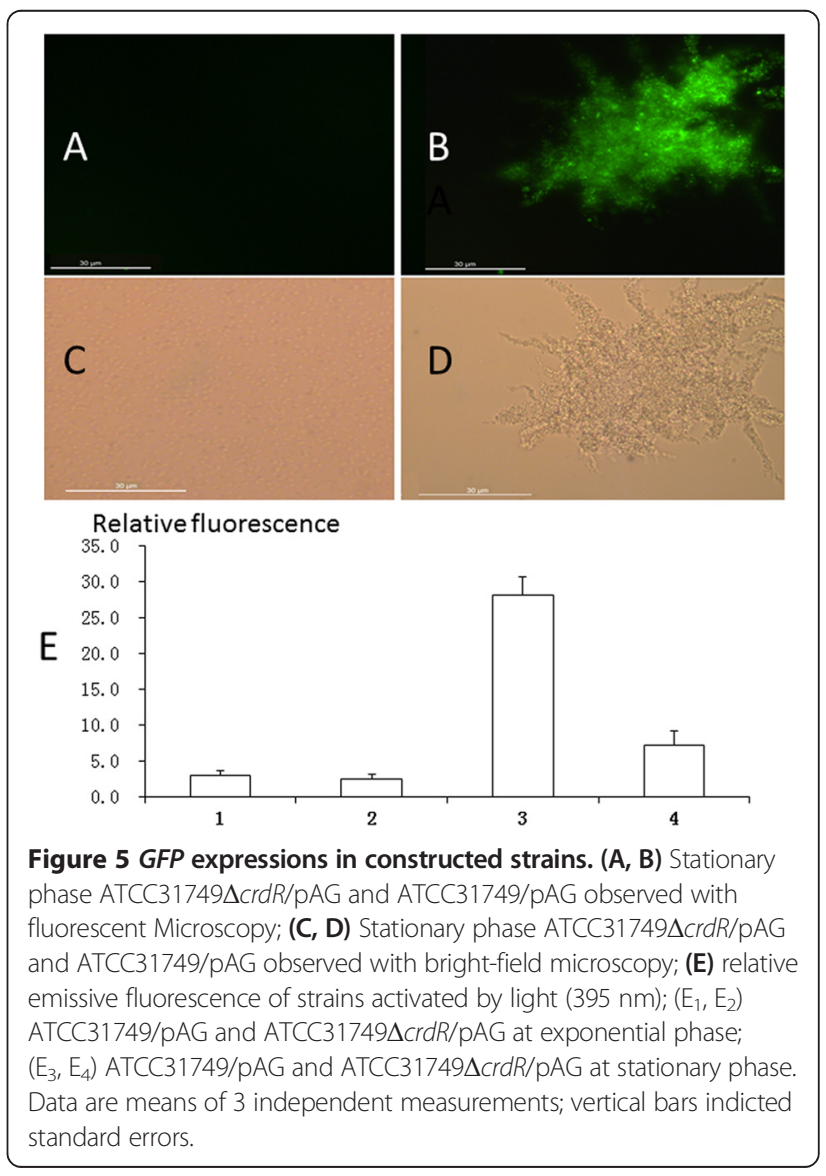




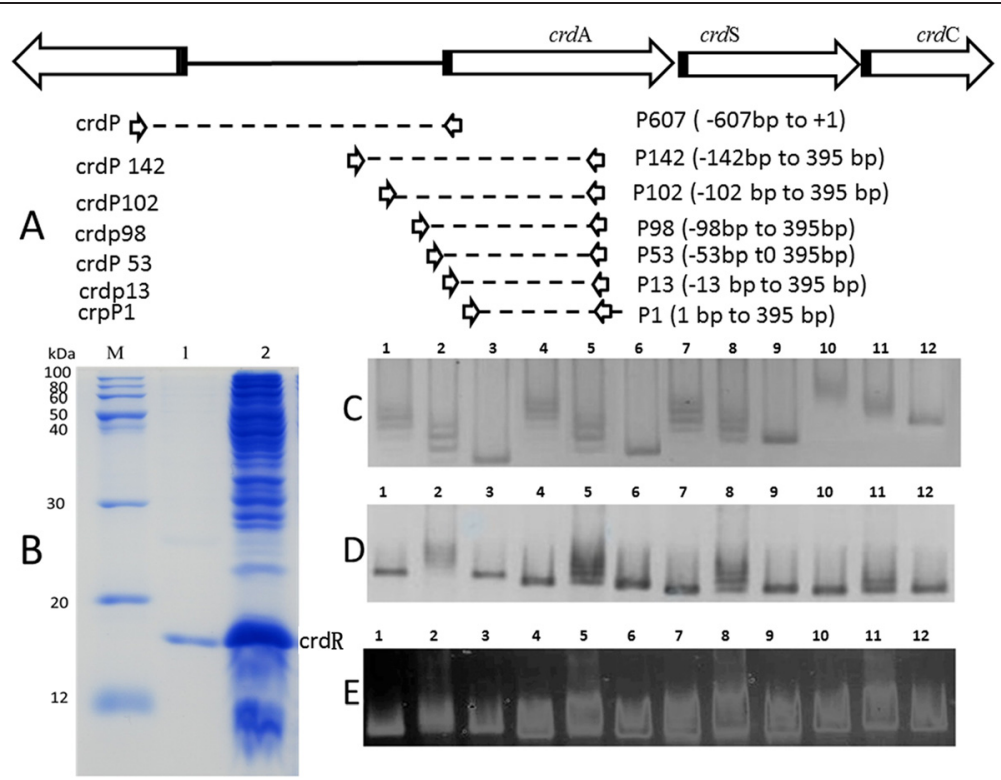

Figure 6 Binding ability of 6 His-tagged crdR to different DNA fragments. A: Different region of the crdP; B: purification of 6 His-tagged crdR; C, D: Binding ability 6 His-tagged crdR to different regions of crdP; E: Binding of 6 His-tagged crdR, to 450 bp upstream of ATG at different gene coding regions. $B_{M}$ : protein markers, $B_{1}$ : purified His-tagged crdR, $B_{2}$ : supernatant of pBQcrdR/E. coli Bl21; $C_{1}$. $C_{4}, C_{7}$, and $C_{10}$ are $10 \mu \mathrm{L}$ of $0.5 \mu \mathrm{M}$ crdP 98, crdP102, crdP142 and crdP mixed with $10 \mathrm{~L}$ of $0.5 \mathrm{mg} / \mathrm{mL}$ purified 6 His-tagged crdR protein respectively, $C_{2}, C_{5}, C_{8}$, and $C_{11}$ are same as $C_{1}$. $C_{4}, C_{7}$, and $C_{10}$, except 6 His-tagged crdR protein was reduced to $0.25 \mathrm{mg} / \mathrm{mL} ; C_{3}, C_{8}, C_{9}$, and $C_{12}$ are same as $C_{1}$. $C_{4}, C_{7}$, and $C_{10}$, without 6 His-tagged crdR. $D_{1}$. $D_{4}, D_{7}$, and $D_{10}$ are $10 \mu \mathrm{L}$ of $0.5 \mu \mathrm{M} \operatorname{crdP} 98, \operatorname{crdP53}, \operatorname{crdP13}$, and crdP1 only respectively, $\mathrm{D}_{2}, \mathrm{D}_{5}, \mathrm{D}_{8}$, and $\mathrm{D}_{11}$ are same of . $D_{1}$. $D_{4}, D_{7}$, and $D_{10}$ mixed with $10 \mu \mathrm{L} 0.5 \mathrm{mg} / \mathrm{mL} 6$ His-tagged crdR protein; $D_{3}, D_{8}, D_{9}$, and $D_{12}$ are same of . $D_{1}, D_{4}, D_{7}$, and $D_{10}$ mixed with $10 \mu \mathrm{L} 0.5 \mathrm{mg} / \mathrm{mL}$ BSA respectively, $E_{1}, E_{4}, E_{7}$, and $E_{10}$ are crdRP, celAP, and $c r d S P, E_{2}, E_{5}, E_{8}$, and $E_{11}$ are same of $E_{1}, E_{4}, E_{7}$, and $E_{10}$ mixed with $10 \mu \mathrm{L}$ of $0.25 \mathrm{mg} / \mathrm{mL} 6$ His-tagged crdR protein respectively, $E_{3}, E_{6}, E_{9}$, and $E_{12}$ are same of $E_{1}, E_{4}, E_{7}$, and $E_{10}$ mixed with $10 \mu \mathrm{L} \mathrm{of} 0.5 \mathrm{mg} / \mathrm{mL}$ $\mathrm{BSA}$ respectively.

and -13 regions of the $c r d$ promoter mixed with or without His-tagged crdR were observed and $-98,-53$ and -13 could all bind to $c r d R$. However the greatest gel mobility shift was observed with the -98 fragment of $c r d P$.

\section{Discussion}

Here, we report that the $c r d R$, a homolog of $P h r R$ of $S$. meliloti can activate curdlan synthetic gene expression in Agrobaterium sp. ATCC31749. To our knowledge, ours is the first report to depict molecular functions of the $c r d R$ gene. Our data indicate that curdlan yield in an over-expressing $c r d R$ strain increased 38\% compared to the WT strain. Also, pBQNcrdR transformed to the $c r d R$ mutant strain recovered 105\% curdlan synthesis of the WT strain (Figure 4). Also, when pAG was transformed into both $c r d R$ mutant and WT strains GFP expression controlled by the $c r d$ promoter was undetectable by fluorescent microscopy with low relative fluorescence in the $\operatorname{crdR}$ mutant. In contrast, the WT strain had visible green color with high relative fluorescence. Finally, q-RT-PCR analysis indicated that $c r d R$ is highly expressed in the stationary phase and that overexpression of $c r d R$ in the WT strain significantly increased expression of $\operatorname{crd} A, \mathrm{crdS}$ and $c r d C$. These data agree with previous reports that $\operatorname{crdR}$ is key for regulating curdlan biosynthesis. Purified
crdR from E. coli BL21 can also specifically bind to the promoter region of $c r d$ offering initial evidence that $\mathrm{crdR}$ is a positive transcriptional regulator of the $\mathrm{crd}$ operon in ATCC31749.

The biomass accumulation in $c r d R$ mutant strains was not significantly different from the WT strain, suggesting that the $c r d R$ gene is not required for cell growth. Microscopic observation revealed that the $c r d R$ mutant was nearly curdlan deficient, resulting in mutant cells with non-capsulated planktonic forms. The WT ATCC31749 strain and the complementary strain of the $c r d R$ mutant, ATCC31749 $\Delta c r d R / \mathrm{pBQN} c r d R$, accumulated curdlan in the stationary phase in culture media with low $\mathrm{pH}$ and limited nitrogen, leading to cells were capsulated and aggregated (Figure 3). In addition, expression of $c r d R$ was higher in the stationary phase than in the exponential phase, and $c r d R$ expression further activated curdlan biosynthesis in the ATCC31749 strain to generate a biofilm. This suggests that curdlan may be critical for biofilm formation in ATCC31749 for improving stress tolerance to harsh conditions.

Bioinformatic analysis indicated that crdR can be grouped into a conserved XRE-family of transcriptional factors that is comprised of HipB in E. coli, PhrR in S. meliloti, CH00371 in R. etli and PraR in A. caulinodans (Additional 
file 1) [27]. Apparently, diverse stress can induce expressions of XRE-family transcriptional factors. Combining with HipA, HipB, a crdR homologue of E. coli that mediates multidrug stress tolerance can bind to its cis elements with conserved sequences of TATCCN ${ }_{8}$ GGATA (where $\mathrm{N}_{8}$ indicates any 8 nucleotides). Genomic scanning indicated that there is no HipA counterpart in the ATCC31749 strain, and that there were no conservative TATCCN8GGATA sequences in the promoter region of the $\mathrm{crd}$ operon. However, $c r d P$ does have three distinct hairpin structures located at the $-10,-35$, and -92 regions (Additional file 2), which are putative crdR binding sites. That purified crdR can bind to an amplified fragment containing the -92 region of $c r d P$ more than the -53 , and -13 regions indicates that those region are likely the crdR binding site. HipAB is a heterodimer of a transcriptional repressive regulator [28], and crdR may play a different role as a transcriptional activator in the form of a homotetramer or homodimer, which must be confirmed with additional studies. PhrR in S. meliloti was affected by low $\mathrm{pH}, \mathrm{Cu}^{2}{ }^{+}, \mathrm{Zn}^{2}{ }^{+}$and $\mathrm{H}_{2} \mathrm{O}_{2}$ stresses [26]. Expression of $\mathrm{CHOO371}$ in $\mathrm{R}$. etli was promoted by oxidation and osmotic shock [29,30]; whereas expression of PraR in A. caulinodans was increased by low nitrogen [31]. Primary data from transcriptome analysis obtained from RNAseq indicates that expression of $c r d R$ in the curdlan fermenting phase with both low $\mathrm{pH}$ and limited nitrogen was twice as great as that in the growing phase of bacterium in LB medium (data not shown) and q-RTPCR analysis of $c r d R$ expression agreed with RNA-Seq data (Table 3). Thus, $c r d R$ expression should be triggered by stress factors as well.

Most organisms within the genera of Rhizobium, Azorhizobium, Bradyrhizobium, Mesorhizobium, and Sinorhizobium are symbiotic bacteria to various leguminous plants [32,33]. After bacteria enter plant tissues, their environment changes to low $\mathrm{pH}$ (normally 5.5), with limited nitrogen and sufficient carbohydrates [32,33], and this environmental shift affects strain morphology and physiology. To survive with limited nitrogen, genes related to bacterial nitrogen fixing and those for low $\mathrm{pH}$ tolerance are expressed. Transcriptional factors such as $\mathrm{PhrR}$ and its homologues are instrumental for expression of low pH tolerance genes. Also, with abundant carbohydrates from host photosynthesis, bacterial extracellular polysaccharides (EPS) are synthesized to produce a biofilm [34] to protect the bacteria from stress. Also, the biofilm provides a low-oxygen environment inside the bacteria to support nitrogen fixing reactions. This concept is in agreement with research with $\operatorname{PraR}$ from $A$. caulinodans ORS571, a homolog of $c r d R$, which can mediate stem nodule formation, regulate expression of $R e b$ genes, and increase nitrogen fixation of bacterial strains within stem nodules [31]. Therefore, biosynthesis of curdlan regulated by $c r d R$ may originate from ancestral characteristics for survival within a host plant. Currently, detailed regulatory mechanisms of $c r d R$ expression, controlled by disadvantageous conditions such as low $\mathrm{pH}$ and limited nitrogen remain unknown. However, reports regarding stringent response signal(p)ppGpp, which can induce EPS biosynthesis and bacterial biofilm formation [35] is worthy of study. We hypothesize that $c r d R$ expression may be activated by (p)ppGpp, which accumulates under stressful conditions.

\section{Conclusions}

In this study, we confirmed that crdR regulates curdlan synthesis by activating expressions of its biosynthetic genes. Ours is the first work to identify XER family transcriptional factor which can activate EPS biosynthesis. crdR may be a multiple-effect regulator controlling expression(s) of the curdlan synthesis gene(s) in ATCC31749 under oxidative stress/low $\mathrm{pH}$ and/or limited nitrogen with abundant sugar. This function of curdlan regulation indicates that curdlan biosynthesis of ATCC31749 under harsh conditions may have evolutionary origin.

\section{Additional files}

\section{Additional file 1: Comparative analysis of crdR homologous protein. Protein sequences of $\mathrm{crdR}$ homologous were compared clustalX2 software and PhrR (with 90\% similarity to crdR, the same as below) from S. meliloti, CH00371 (86\%) from R. leguminosarum, PraR (57\%) from A. Caulinodans. \\ Additional file 2: A98 pb promoter sequence of the $c r d$ operon ( $c r d P$ ) upstream from starting codon of $c r d A$ (ATG) with its secondary structure predicted by DNAMAN 98 bp fragment upstream ATG) of the $c r d A$ have 3 putative crdR binding sites, which has 3 hairpin structures located in $-92,-35$, and -10 regions.}

Authors' contributions

$X Y$ constructed vectors $\mathrm{pAG}$ and $\mathrm{pBQ}-\mathrm{crdR}$, analyzed curdlan yield and measured GFP expression, performed q-RT-PCR analysis, crdR purification, and EMSA and drafted the manuscript. CZ constructed vectors of pUC19T-crdR and $\mathrm{pBQN} c r d R$, assisted with $c r d R$ knockout and edited the paper. LY assisted with crdR knockout. LZ assisted with statistics and bioinformatic analysis. CL assisted with curdlan yield analysis. ZL assisted with experimental conditions and manuscript editing. ZM conceptualized the study and coordinated the study design as well as assisted with the paper. All authors read and approved the final manuscript.

\section{Acknowledgements}

The study was supported by the Chinese Natural Science Foundation (No. 31170057) and the Yunnan Natural Science Foundation (No. 2010CD054).

Received: 6 October 2014 Accepted: 20 January 2015

Published online: 10 February 2015

References

1. Sutherland IW. Microbial polysaccharides from Gram-negative bacteria. Int Dairy J. 2001;11(9):663-74.

2. Harada T, Yoshimura T. Production of a new acidic polysaccharide containing succinic acid by a soil bacterium. Biochim Biophys Acta (BBA). 1964;83(3):374-6.

3. Kim MK, Lee IY, Ko JH, Rhee YH, Park YH. Higher intracellular levels of uridinemonophosphate under nitrogen-limited conditions enhance 
metabolic flux of curdlan synthesis in Agrobacterium Species. Biotechnol Bioeng. 1999;62(3):317-23.

4. Zhang H, Nishinari K, Williams MA, Foster TJ, Norton IT. A molecular description of the gelation mechanism of curdlan. Int J Biol Macromol 2002;30(1):7-16.

5. Lehtovaara BC, Gu FX. Pharmacological, structural, and drug delivery properties and applications of 1,3- $\beta$-glucans. J Agric Food Chem. 2011;59(13):6813-28.

6. Zhan X-B, Lin C-C, Zhang H-T. Recent advances in curdlan biosynthesis, biotechnological production, and applications. Appl Microbiol Biotechnol. 2012;93(2):525-31.

7. Popescu I, Pelin IM, Butnaru M, Fundueanu G, Suflet DM. Phosphorylated curdlan microgels. Preparation, characterization, and in vitro drug release studies. Carbohydr poly. 2013;94(2):889-98.

8. Reese ET, Mandels M. $\beta$-D-1,3 glucanases in fungi. Can J microbiol. 1959:5(2):173-85.

9. Hoffmann G, Timell T. Isolation of a $\beta-1$, 3-glucan (laricinan) from compression wood of Larix laricina. Wood Sci Technol. 1970;4(2):159-62.

10. Zheng Z-Y, Lee JW, Zhan XB, Shi Z, Wang L, Zhu L, et al. Effect of metabolic structures and energy requirements on curdlan production by Alcaligenes faecalis. Biotechnol Biopro Eng. 2007;12(4):359-65.

11. Footrakul P, Suyanandana P, Amemura A, Harada T. Extracellular polysaccharides of Rhizobium from the Bangkok MIRCEN collection. J Ferment Tech. 1981;59(1):9-14.

12. Kenyon W, Buller C. Structural analysis of the curdlan-like exopolysaccharide produced by Cellulomonas flavigena KU. J Ind Microbiol Biotechnol. 2002;29(4):200-3.

13. Goodner B, Hinkle G, Gattung S, Miller N, Blanchard M, Qurollo B, et al. Genome sequence of the plant pathogen and biotechnology agent Agrobacterium tumefaciens C58. Science. 2001;294(5550):2323-8.

14. Ruffing AM, Castro-Melchor M, Hu W-S, Chen RR. Genome sequence of the curdlan-producing Agrobacterium sp. strain ATCC 31749. J bacteriol. 2011;193(16):4294-5.

15. Stasinopoulos SJ, Fisher PR, Stone BA, Stanisich VA. Detection of two loc involved in ( $1 \rightarrow 3$ )- $\beta$-glucan (curdlan) biosynthesis by Agrobacterium sp. ATCC31749, and comparative sequence analysis of the putative curdlan synthase gene. Glycobiology. 1999;9(1):31-41.

16. Karnezis T, Epa VC, Stone BA, Stanisich VA. Topological characterization of an inner membrane (1 $\rightarrow 3$ )- $\beta$-D-glucan (curdlan) synthase from Agrobacterium sp. strain ATCC31749. Glycobiology. 2003;13(10):693-706.

17. Hrmova M, Stone BA, Fincher GB. High-yield production, refolding and a molecular modelling of the catalytic module of (1,3)-- $\beta$-d-glucan (curdlan) synthase from Agrobacterium sp. Glycoconj J. 2010;27(4):461-76.

18. Jin L-H, Um H-J, Yin C-J, Kim Y-H, Lee J-H. Proteomic analysis of curdlan-producing Agrobacterium sp. in response to $\mathrm{pH}$ downshift. J Biotechnol. 2008;138(3):80-7.

19. Jiang L. Effect of nitrogen source on curdlan production by Alcaligenes faecalis ATCC 31749. Int J Biol Macromol. 2013;21(2):218-20.

20. Zhang H-T, Zhan X-B, Zheng Z-Y, Wu J-R, English N, Yu X-B, et al. Improved curdlan fermentation process based on optimization of dissolved oxygen combined with $\mathrm{pH}$ control and metabolic characterization of Agrobacterium sp. ATCC 31749. Appl Microbiol Biotechnol. 2012;93(1):367-79.

21. J-h L, Lee IY. Optimization of uracil addition for curdlan ( $\beta-1 \rightarrow 3$-glucan) production by Agrobacterium sp. Biotechnol Lett. 2001;23(14):1131-4.

22. Yu L, Wu J, Liu J, Zhan X, Zheng Z, Lin CC. Enhanced curdlan production in Agrobacterium sp. ATCC 31749 by addition of low-polyphosphates. Biotechnol Biopro Eng. 2011;16(1):34-41.

23. West T-P. Pyrimidine base supplementation effects curdlan production in Agrobacterium sp. ATCC31749. J Basic Microbiol. 2006;46(2):153-7.

24. Ruffing AM, Chen RR. Transcriptome profiling of a curdlan-producing Agrobacterium reveals conserved regulatory mechanisms of exopolysaccharide biosynthesis. Microb Cell Fact. 2012;11(1):1-13.

25. Galibert F, Finan TM, Long SR, Pühler A, Abola P, Ampe F, et al. The composite genome of the legume symbiont Sinorhizobium meliloti Science. 2001;293(5530):668-72.

26. Reeve WG, Tiwari RP, Wong CM, Dilworth MJ, Glenn AR. The transcriptional regulator gene phrR in Sinorhizobium meliloti WSM419 is regulated by low pH and other stresses. Microbiology. 1998;144(12):3335-42.

27. Lin C-Y, Awano N, Masuda H, Park J-H, Inouye M. Transcriptional repressor HipB regulates the multiple promoters in Escherichia coli. J Mol Microbiol Biotechnol. 2013;23(6):440-7.
28. Schumacher MA, Piro KM, Xu W, Hansen S, Lewis K, Brennan RG. Molecular mechanisms of HipA-mediated multidrug tolerance and its neutralization by HipB. Science. 2009;323(5912):396-401.

29. Vercruysse M, Fauvart M, Jans A, Beullens S, Braeken K, Cloots L, et al. Stress response regulators identified through genome-wide transcriptome analysis of the (p) ppGpp-dependent response in Rhizobium etli. Genome Biol. 2011;12(2):R17.

30. Martínez-Salazar JM, Salazar E, Encarnación S, Ramírez-Romero MA, Rivera J. Role of the extracytoplasmic function sigma factor RpoE4 in oxidative and osmotic stress responses in Rhizobium etli. J Bacteriol. 2009;191(13):4122-32.

31. Akiba N, Aono T, Toyazaki H, Sato S, Oyaizu H. phrR-like gene praR of Azorhizobium caulinodans ORS571 is essential for symbiosis with Sesbania rostrata and is involved in expression of reb genes. Appl Environ Microbiol. 2010;76(11):3475-85.

32. Raven J, Smith F. Nitrogen assimilation and transport in vascular land plants in relation to intracellular pH regulation. New Phytologist. 1976;76(3):415-31.

33. Smith FA, Raven JA. Intracellular pH and its regulation. Annu Rev Plant Physiol. 1979;30(1):289-311.

34. Flemming H-C, Neu TR, Wozniak DJ. The EPS matrix: the "house of biofilm cells". J Bacteriol. 2007;189(22):7945-7.

35. Potrykus K, Cashel M. (p) ppGpp: Still magical? Annu Rev Microbiol. 2008;62:35-51.

36. Yu L, Wu J, Zheng Z, Lin C, Zhan X. Changes in gene transcription and protein expression involved in the response of Agrobacterium sp. ATCC 31749 to nitrogen availability during curdlan production. Prikl Biokhim Mikrobiol. 2011:47(5):537-43.

37. Hoang TT, Karkhoff-Schweizer RR, Kutchma AJ, Schweizer HP. A broad-hostrange Flp-FRT recombination system for site-specific excision of chromosomally-located DNA sequences: application for isolation of unmarked Pseudomonas aeruginosa mutants. Gene. 1998;212(1):77-86.

38. Mao Z, Chen RR. Recombinant synthesis of hyaluronan by Agrobacterium sp. Biotechnol Prog. 2007;23(5):1038-42.

39. Toyoda K, Teramoto H, Inui M, Yukawa H. Expression of the gapA gene encoding glyceraldehyde-3-phosphate dehydrogenase of Corynebacterium glutamicum is regulated by the global regulator SugR. Appl Microbiol Biotechnol. 2008:81(2):291-301.

40. Parés-Matos El. Electrophoretic mobility-shift and super-shift assays for studies and characterization of protein-DNA complexes. Methods Mol Biol. 2013;977:159-67.

\section{Submit your next manuscript to BioMed Central and take full advantage of:}

- Convenient online submission

- Thorough peer review

- No space constraints or color figure charges

- Immediate publication on acceptance

- Inclusion in PubMed, CAS, Scopus and Google Scholar

- Research which is freely available for redistribution 